\title{
Relación entre psicopatologías e ideación suicida en adolescentes escolarizados de Colombia
}

\section{Relationship between psychopathologies and suicidal ideation in school adolescents in Colombia}

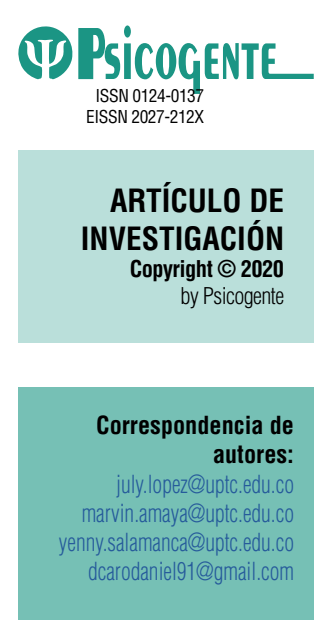

Recibido: 5-02-20 Aceptado: $15-05-20$ Publicado: $30-06-20$

\author{
July Marcela López-Vega (D) - Marvin Katherine Amaya-Gil (D) - Yenny \\ Salamanca-Camargo (iD - José Daniel Caro-Castillo (iD \\ Universidad Pedagógica y Tecnológica de Colombia, Tunja, Colombia
}

Resumen:

Introducción: Según los estudios, la adolescencia es un periodo de alta vulnerabilidad para el desarrollo de psicopatologías y aparición de conductas suicidas, siendo cada vez más evidente su presencia y aumento, a menor edad.

Objetivo: analizar la relación entre psicopatologías e ideación suicida en adolescentes escolarizados.

Método: Estudio no experimental de tipo descriptivo correlacional y corte transversal, a partir del Inventario de Evaluación de Personalidad para Adolescentes y el Inventario de Ideación Suicida Positiva y Negativa, en una muestra de 249 adolescentes (134 mujeres y 115 hombres), con edades entre 12 y 17 años y escolaridad entre sexto y undécimo grado, seleccionados por muestreo estratificado.

Resultados: Los análisis descriptivos y factoriales revelan mayor presencia de síntomas psicopatológicos como quejas somáticas y manía (12,4\%), esquizofrenia y trastornos de ansiedad $(8,8 \%)$, y depresión (7,6 \%); así mismo, se reportó ideación suicida entre niveles medio y alto en un $48,5 \%$; respecto a la correlación, el análisis factorial exploratorio arrojó dos factores: el primero, con las escalas esquizofrenia $(0,76)$, ansiedad $(0,67)$, trastornos relacionados con ansiedad $(0,66)$, paranoia $(0,65)$, somatización $(0,60)$, rasgos límites $(0,47)$, depresión $(0,46)$ y manía $(0,43)$, relacionaron con ideación suicida positiva $(0,24)$; y el segundo, las escalas depresión $(0,46)$ y rasgos límites $(0,47)$, con ideación suicida negativa $(0,79)$.

Conclusiones: Si bien, se identificó la relación entre las psicopatologías reportadas y la ideación suicida, depresión y rasgos límites, correlacionan con pensamientos suicidas; las restantes, con déficit de factores protectores.

Palabras clave: salud mental, enfermedades mentales, suicidio.

Abstract:

Introduction: According to studies, adolescence is a period of high vulnerability for the development of psychopathologies and the appearance of suicidal behavior, its presence and increase becoming increasingly evident at a younger age.

Objective: Was to analyze the relationship between psychopathology and suicidal ideation in adolescents schooling.

Method: Non-experimental study a correlational descriptive, from the Adolescent Personality Assessment Inventory and the Positive and Negative Suicidal Ideation Inventory. 249 adolescents, (134 women and 115 men), with ages between 12 and 17 years and schooling between the sixth and eleventh grade selected by stratified sampling.

Results: Descriptive and factorial analyses reveal greater presence of psychopathological symptoms related to somatic complaints and mania $(12,4 \%)$, schizophrenia and anxiety-related disorders $(8,8 \%)$, and depression (7,6 \%); suicide ideation was reported between medium and high levels in $48,5 \%$ of participants. With respect to correlation, exploratory factorial analysis yields two factors: the first, includes schizophrenia scales $(0,76)$, anxiety $(0,67)$, anxiety-related disorders $(0,66)$, paranoia $(0,65)$, somatization $(0,60)$, boundary traits $(0,47)$, depression $(0,46)$ and mania $(0,43)$ and is related to positive suicidal ideation $(0,24)$; and the second, includes depression scales $(0,46)$ and limiting traits $(0,47)$ and

Cómo citar este artículo (APA):

Lopez-Vega, J., Amaya-Gil, M. K., Salamanca Camargo, Y. \& Caro Castillo, J. D. (2020). Relación entre psicopatologías e ideación suicida en adolescentes escolarizados de Colombia. Psicogente 23(44), 1-18. https://doi.org/10.17081/psico.23.44.3709 
are associated with negative suicidal ideation $(0,79)$.

Conclusion: that while the psychopathologies evaluated are related to suicidal ideation, only the pathologies of depression and limiting traits correlate with suicidal thoughts, specifically, the others, are related to the deficiency of protective factors.

Keywords: mental health, psychopathology, suicidal ideation.

\section{INTRODUCCIÓN}

La Organización Mundial de la Salud (2018), refiere que la adolescencia es un periodo de alta vulnerabilidad tanto para el desarrollo de psicopatologías como para el riesgo de conducta suicida debido a cambios abruptos relacionados con mayor autonomía, pertenencia a un grupo, exploración de identidad sexual, mayor acceso y uso a la tecnología, violencia, problemas socioeconómicos, entornos vulnerables y fuentes de apoyo débiles; de igual manera, indica que a nivel mundial prevalecen los trastornos relacionados con depresión (tasa de 300 millones de casos), afectivo bipolar (60 millones), demencia (47,5 millones) y esquizofrenia ( 21 millones). En Colombia, el Ministerio de Salud (2018) registró para el año 2016 una tasa de mortalidad de 1,53 por 100.000 personas, debido a la presencia de alguna psicopatología. El acto suicida por su parte, ha sido considerado como un problema mundial de salud pública, presentándose alrededor de 800.000 casos al año (OMS, 2017); en este mismo año, el Instituto Nacional de Medicina Legal y Ciencias Forenses (INMLCF, 2018), reportó que en Colombia ha sido considerada como la cuarta causa de muerte $(10,53 \%)$, después del homicidio $(46,90 \%)$, accidente de transporte $(28,8 \%)$ y muerte accidental $(14,49 \%)$ y específicamente, en 2018 , se registraron 2.464 casos de los cuales, 2.026 $(82,2 \%)$ eran hombres y $438(17,7 \%)$ mujeres, siendo más frecuente entre los 20 y 24 años.

El fenómeno del suicidio, ha sido abordado desde la psicología como un espectro conformado por tres fases: ideación suicida, intento suicida y suicidio consumado; para el presente estudio, se tuvo en cuenta la primera fase, en tanto que como lo ha expuesto Gómez (2012), tiene un carácter predictivo en el comportamiento suicida y además, según Nock, Borges, Bromet, Alonso, Angermeyer, Beautrais, Bruffaerts, Chiu, De Girolamo, Gluzman, De Graaf, Gureje, Haro, Huang, Karam, Kessler, Lepine, Levinson, Medina-Mora, Ono, Posada-Villa y Williams (2008), se ha relacionado con el aumento del riesgo suicida durante el primer año desde su aparición; siendo esencial su abordaje como estrategia de prevención (Rosales, 2010). Dentro de los antecedentes empíricos relacionados con adolescentes latinoamericanos, se resaltan los estudios de Rodríguez, Rincón, Velasco, Hernández, Ramos y González (2012), 
quienes reportaron que de 254 pacientes, el $49 \%$ manifestaba ideación suicida, siendo más prevalente en la adolescencia; Flores, Perales y Gómez (2016), en su estudio con 260 adolescentes mexicanos, encontraron en un 33 \% manifestaciones de ideación suicida; Varengo (2016), identificó que el $37 \%$ de 96 participantes argentinos, reportaron niveles moderado y alto de ideación suicida; Pérez y Salamanca (2017), refieren la presencia de esta manifestación en 37 de los 76 participantes vinculados, y Forero, Siabato y Salamanca (2017), reportaron dicha manifestación en el 30 \% de los 289 participantes.

Por su parte, Bedoya y Montaño (2016), han referido que los trastornos mentales constituyen un factor de riesgo relevante para la conducta suicida, y que hasta en el $90 \%$ de los casos de suicidio, se ha presentado algún trastorno mental asociado, siendo la depresión y la esquizofrenia, las de mayor riesgo. En cuanto a la relación entre estas variables, se encontró que la depresión, consumo de tóxicos (alcohol, opiáceos y estimulantes), trastornos de personalidad, enfermedades somáticas (dolor crónico o intenso), esquizofrenia y trastorno bipolar; así como las dimensiones psicológicas impulsividad, agresividad, desesperanza y rigidez cognitiva, son factores de riesgo que conllevan al suicidio (Bedoya y Montaño, 2016; Cabra, Infante y Sossa, 2010; GonzálezRodríguez, Molina-Andreu, Navarro, Gastó, Penadés y Catalán, 2014; Ridge, Keyes \& Jobes, 2016; Nizama, 2011; Toledo, 2012).

Adicionalmente, Carvajal y Caro (2011), encontraron que el consumo de sustancias psicoactivas y la depresión, así como el antecedente de intento de suicidio, baja autoestima y pertenecer a una familia poco saludable, explican en gran medida la presencia de ideación suicida; Ceballos, Suárez, Suescún, Gamarra, González y Sotelo (2015) en su estudio con 242 adolescentes de Santa Marta, encontraron que mejor autoestima y baja depresión, es un indicador de menor probabilidad de ideación suicida

Teniendo en cuenta estos antecedentes, si bien, se identifican algunas investigaciones dedicadas al estudio de la relación entre distintos trastornos psicológicos y el comportamiento suicida; a nivel nacional, los estudios se centran en especial en los trastornos relacionados con el estado de ánimo y el consumo de sustancias psicoactivas, dejando de lado otras psicopatologías que también pueden estar relacionadas. Esta investigación, pretende analizar el comportamiento entre los factores protectores y de riesgo de ideación suicida, y la presencia de diferentes psicopatologías, a partir del uso de modelos de ecuaciones estructurales, como el Análisis Factorial Exploratorio - AFE y el Análisis Factorial Confirmatorio AFC, que por ser técnicas de 
reducción de datos, permiten explicar de mejor forma la correlación entre variables observadas, y a su vez, busca establecer condiciones predictivas de la ideación suicida; sus hallazgos, aportan evidencia empírica en aspectos como la importancia del uso de instrumentos que cuenten con buenas propiedades psicométricas, el estado de la salud mental de los adolescentes colombianos y el apoyo en la evaluación de diferentes manifestaciones psicopatológicas, como insumo, para la generación de propuestas de intervención a nivel primario, secundario y terciario, tanto a nivel individual, como grupal.

\section{METOdologíA}

\subsection{Diseño}

Estudio no experimental, de tipo descriptivo correlacional y corte transversal (Hernández, Fernández- Collado \& Baptista, 2010).

\subsection{Participantes}

Del total de participantes correspondiente a 2.474 adolescentes, se tomó una muestra de 249 participantes. El tamaño de la muestra fue calculado mediante la fórmula para muestras representativas con población finita, obteniendo un tamaño de muestra mínimo, de 134 (53,8 \%) mujeres y 115 $(46,2 \%)$ hombres; asimismo, con las cifras suministradas por las Instituciones vinculadas, se realizó un muestreo vía estratificación a fijación proporcional al tamaño, por edades desde los 12 a los 17, donde cada edad correspondió a un estrato, con la finalidad de que la muestra se distribuyera de acuerdo a las características de la población.

\subsection{Instrumentos}

\subsubsection{Inventario de Evaluación de la Personalidad para Adolescentes} (PAI-A, Personality Assessment Inventory-Adolescent)

Diseñado por Morey (2007, adaptado a la lengua española por Cardenal, OrtizTallo y Santamaría, 2012 y Baremado para Colombia por Vásquez, Galván, Guerrero, Simancas, Romero-Acosta, Ramírez, Yañez, Ferrel, Ferrel, Galván y Salamanca, 2018). Se trata de un instrumento que cuenta con una confiabilidad del 0,82 y permite evaluar de forma amplia aspectos relacionados con la personalidad e identificar alguna psicopatología, mediante 264 ítems agrupados en 22 escalas, divididas de la siguiente manera, cuatro escalas de validez (Inconsistencia, Infrecuencia, Impresión negativa e Impresión positiva); 11 escalas clínicas (Quejas somáticas, Ansiedad, Trastornos relacionados con la ansiedad, Depresión, Manía, Paranoia, Esquizofrenia, Rasgos 
Límites, Rasgos antisociales, Problemas con el alcohol y Problemas con las drogas); cinco escalas de consideraciones para el tratamiento (Agresión, Ideaciones suicidas, Estrés, Falta de apoyo social y Rechazo al tratamiento), y dos escalas de relaciones interpersonales (Dominancia y Afabilidad); las cuales, se miden mediante una escala tipo likert: falso, ligeramente verdadero, bastante verdadero y completamente verdadero.

\subsubsection{Inventario de Ideación Suicida Positiva y Negativa (PANSI, Positive} and Negative Suicidal Ideation)

Elaborado por Osman, Gutiérrez, Kopper, Barrios, \& Chiro (1998 y adaptado y validado para Colombia por Villalobos-Galvis, 2009). Este instrumento, cuenta con un alpha de Cronbach de 0,899 y permite identificar el riesgo de suicidio, a partir de la evaluación de la Ideación Suicida - IS, con 14 ítems, siendo 6 ítems de Ideación Suicida Positiva - ISP, que corresponde a los factores protectores y son puntuados de manera inversa; y 8 ítems de Ideación Suicida Negativa - ISN, relacionados con factores de riesgo. Cada ítem consta de cinco opciones de respuesta tipo likert (nunca, rara vez, algunas veces, casi siempre y siempre) que deben ser seleccionadas teniendo en cuenta las últimas dos semanas.

\subsection{Procedimiento}

Inicialmente se solicitó el aval de dos Instituciones Educativas Públicas para la participación en el presente estudio, luego se informó sobre el objetivo de la investigación, el tratamiento de los resultados y demás aspectos éticos relacionados, tanto a profesores, como a padres y estudiantes y se les invitó a participar de manera voluntaria y respaldados por un consentimiento informado por parte de los padres y un asentimiento informado por parte de los participantes. Una vez obtenida la autorización, se realizó la aplicación de los instrumentos en compañía del docente orientador en las instalaciones de cada Institución. La información recopilada fue analizada mediante el software libre $R$ stadistic y los hallazgos fueron contrastados de acuerdo a la revisión teórico-empírica realizada.

\subsection{Análisis de datos}

Se realizó un análisis descriptivo de datos, usando medidas de tendencia central y dispersión, cuadros de resumen de frecuencias y tablas de contingencia que permitieron conocer las características principales de las 
mediciones resultantes en la aplicación de los instrumentos. Con el fin de determinar la viabilidad del Análisis Factorial (AF) se revisó el coeficiente de adecuación de muestras - KMO, la cual presentó un valor de 0,889 , indicando su aplicabilidad; asimismo, se usó la técnica de Análisis de Componentes Principales $(A C P)$ para extraer los componentes suficientes para explicar la variabilidad de los datos a partir del método Kaiser. Posteriormente, se realizó un $A F$ Exploratorio, con el fin de evaluar relaciones entre variables estimando la suficiencia de los factores obtenidos mediante la prueba $c^{2} i^{2}(p \leq 0,05)$ y se procedió a usar la técnica de Análisis Factorial Confirmatorio ( $A F C$ ) para evaluar las conclusiones derivadas del AFE, teniendo en cuenta los indicadores $R M S A(\leq 0,05)$, los cuales hacen referencia a la raíz del error cuadrático medio de aproximación y se puede explicar, como el error de aproximación promedio por cada grado de libertad, que para esta investigación, genera valores por debajo de 0,05 lo que indica, un buen ajuste; además se midieron los indicadores de Raíz del Residuo Medio Estandarizado SRMR ( $\leq 8)$ y el índice de ajuste comparativo $\mathrm{CFI}(\approx 1)$.

\section{RESULTADOS}

En primer lugar, se presentan los resultados encontrados para cada una de las 11 psicopatologías evaluadas a partir del PAI-A (Quejas somáticas - SOM; Ansiedad - ANS; Trastornos relacionados con la ansiedad - TRA; Depresión DEP; Manía-MAN; Paranoia-PAR; Esquizofrenia-ESQ; Rasgos Límites-LIM; Rasgos antisociales- ANT; Problemas con el alcohol - ALC y Problemas con las drogas - DRG) y los niveles de ideación suicida (ideación suicida positiva - ISP, negativa - ISN y global - IS), haciendo énfasis en los niveles medio y alto, los cuales evidencian sintomatología asociada a los dos fenómenos; y en segundo lugar, se muestran las relaciones obtenidas entre las variables objeto de estudio, a partir de los análisis factoriales realizados.

Respecto a las psicopatologías, los hallazgos indican que la mayoría de los participantes se ubicaron en los niveles de bajo y sin síntomas; las escalas clínicas que predominaron en los participantes fueron: quejas somáticas (SOM) y manía (MAN) con $12,4 \%$ cada una, esquizofrenia (ESQ) y trastornos relacionados con la ansiedad (TRA) 8,8 \%, y depresión (DEP) (Tabla 1); cabe aclarar que se omitió el análisis de las cinco escalas relacionadas con consideraciones para el tratamiento y las dos escalas sobre relaciones interpersonales, debido a que no se contemplaron dentro de los objetivos del presente estudio (Véase descripción del instrumento PAI-A). 
Tabla 1

Distribución en escalas PAI-A

\begin{tabular}{ccccc}
\hline & SIN SíNTOMAS & BAJO & MEDIO & ALTO \\
\hline Quejas somáticas & $68,3 \%$ & $19,3 \%$ & $10 \%$ & $10 \%$ \\
Ansiedad & $74,3 \%$ & $19,3 \%$ & $4,8 \%$ & $4,8 \%$ \\
Trastornos relacionados & $67,9 \%$ & $23,3 \%$ & $8 \%$ & $8 \%$ \\
con ansiedad & $70,3 \%$ & $22,1 \%$ & $7,2 \%$ & $7,2 \%$ \\
Depresión & $50 \%$ & $37,3 \%$ & $8 \%$ & $8 \%$ \\
Manía & $72,3 \%$ & $23,3 \%$ & $4 \%$ & $4 \%$ \\
Paranoia & $65,9 \%$ & $25,3 \%$ & $6,8 \%$ & $6,8 \%$ \\
Esquizofrenia & $77,5 \%$ & $17,3 \%$ & $4,8 \%$ & $4,8 \%$ \\
Rasgos Límites & $91,6 \%$ & $8 \%$ & $0,4 \%$ & $0,4 \%$ \\
Rasgos antisociales & $85,5 \%$ & $13,3 \%$ & $0,8 \%$ & $0,8 \%$ \\
Problemas con el alcohol & $79,1 \%$ & 18,1 & $2,8 \%$ & $2,8 \%$ \\
Problemas con las drogas & & & & \\
\hline
\end{tabular}

En cuanto a la edad, los participantes con 13 años presentaron en mayor medida las psicopatologías Trastornos relacionados con la ansiedad, Quejas somáticas (SOM) y Ansiedad (ANS); mientras que los de 16 años presentan síntomas relacionados con Depresión (DEP), Rasgos límites (LIM) y Paranoia (PAR). En relación al sexo, en hombres predominan síntomas relacionados con Manía, (MAN) Esquizofrenia (ESQ), Depresión (DEP), Ansiedad (ANS) y Problemas con el alcohol (ALC); mientras que, en mujeres Quejas somáticas (SOM), Trastornos relacionados con ansiedad (TRA), Paranoia (PAR), Rasgos límites (LIM) y Problemas con las drogas (DRG).

Al analizar la presencia de Ideación Suicida (IS) se identifica que el $30 \%$ de los participantes reportan Ideación Suicida Negativa (ISN), el 52 \% Ideación Suicida Positiva (ISP) y el $48,5 \%$ IS (ver Tabla 2 ) y en cuanto al género, se encontró mayor presencia en mujeres y entre los 12, 13 y 16 años.

Tabla 2

Nivel Ideación Suicida Negativa, Positiva y Total

\begin{tabular}{cccccc}
\hline & MUY BAJO & BAJO & MEDIO & ALTO & MUY ALTO \\
\hline Ideación Suicida Negativa & $69,9 \%$ & 0 & 0 & $22,9 \%$ & $7,2 \%$ \\
Ideación Suicida Positiva & $26,9 \%$ & $21,3 \%$ & $22,9 \%$ & $16,1 \%$ & $12,9 \%$ \\
Ideación Suicida & $24,5 \%$ & $26,9 \%$ & $22,5 \%$ & $19,3 \%$ & 6,8 \\
\hline
\end{tabular}

En el Análisis Factorial Exploratorio, inicialmente, se comprobó el cumplimiento de los supuestos requeridos para su realización (Zamora y Esnaloa, 2015), obteniendo un KMO de 0,889, lo que indica su adecuado uso. Mediante un Análisis de Componentes Principales (ACP), se obtuvieron dos componentes de variabilidad para las escalas clínicas en relación con la IS, atendiendo el método Kaiser que explica que las varianzas $\geq 1$ son candi- 
datos a explicar mejor los datos. El AFE de las escalas clínicas con la Ideación Suicida (IS), arroja dos factores que explican el $41 \%$ de la varianza total, donde el factor 1 tiene mayor influencia con 22,3\% de variabilidad y que solo Depresión (DEP) y Rasgos límites (LIM) se asocian con Ideación Suicida Negativa (ISN) en el factor 2 (ver Tabla 3). Cabe mencionar que las escalas Rasgos antisociales (ANT), Problemas con el alcohol (ALC) y Problemas con las drogas (DRG), no se vincularon a ninguno de los factores, debido a que sus cargas fueron menores al $30 \%$ dentro del factor, y por tanto, no eran significativas para hacer parte del grupo.

Tabla 3

Matriz Análisis Factorial Exploratorio de las escalas PAI-A con sub-escalas PANSI.

\begin{tabular}{llllllllllll}
\hline ESCALAS & SOM & ANS & TRA & DEP & MAN & PAR & ESQ & LIM & ALC & ISN & ISP \\
\hline Factor 1 & 0,539 & 0,592 & 0,698 & 0,476 & 0,566 & 0,592 & 0,756 & 0,495 & 0,351 & & 0,446 \\
Factor 2 & & & & 0,673 & & & & 0,423 & & 0,636 & \\
\hline
\end{tabular}

En cuanto al Análisis Factorial Confirmatorio, se obtuvo un modelo con dos factores que coinciden con el Análisis Factorial Exploratorio (AFE) previamente descrito. Como se observa en la Tabla 3, en el factor latente 1, incluye ocho de las escalas clínicas que se relacionan con la Ideación Suicida Positiva (ISP); mientras que, el factor latente 2 , está compuesto por las escalas Depresión (DEP) y Rasgos Límites (LIM) y relacionadas con la Ideación Suicida Negativa(ISN). Se destaca que las variables Depresión (DEP) y rasgos límites (LIM) son comunes en los dos factores latentes y estos últimos se relacionan entre sí; por su parte, las psicopatologías Problemas con el alcohol (ALC), Problemas con las drogas (DRG) y Rasgos antisociales (ANT), cabe resaltar, que las variables que no generaron puntuación dentro de los factores se descartan, debido a que tienen una puntuación menor del $30 \%$, siendo no significativo para la búsqueda de relaciones y por tanto, no se relacionan en ningún factor. Por su parte, la prueba chi- cuadrado para bondad y ajuste, refleja un estadístico de 148,6 con un $p$ - valor de aproximadamente 0; el Comparativo de ajuste (CFI) con un 0,8 , reflejando que el ajuste del modelo es óptimo, y pese a que el valor RMSEA (Raíz del Error Cuadrático Medio Aproximado ) presenta una puntuación de 0,1, lo cual indica un ajuste débil para el modelo planteado, mientras que, el SRMR (Raíz del Residuo Medio Estandarizado) presenta una puntuación del $8 \%$, indicando un buen ajuste para el modelo planteado.

En la Figura 1, se evidencia la conformación de los factores, de acuerdo a las variables que son significativas para explicar cada uno de estos; para lo 
cual, se considera correlación con el factor, a las puntuaciones que están por encima del 30 \% y que, además, presentan relaciones entre sí.

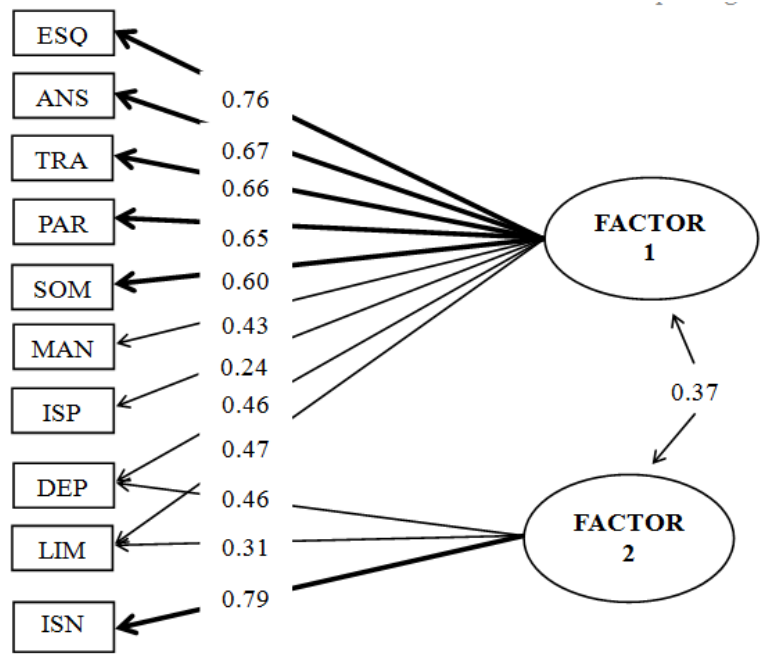

Figura 1. Modelo de dos factores para explicar la relación entre las escalas clínicas e Ideación Suicida

En cuanto a los datos presentados en las figuras, es importante resaltar que, el peso o correlación de cada variable (ítem) con cada factor (matriz factorial), se consideran definidos por los ítems que tienen, en este, los pesos mayores; por tanto, para que un ítem se considere dentro de un factor, o dicho con más propiedad, que lo defina o explique suficientemente, debe tener una correlación razonablemente alta (0,30 o más) y no mayor en otro factor; así mismo, un factor debe estar definido al menos por tres ítems para que merezca la pena tenerlo en cuenta; de esta manera los cálculos que realiza el software $\mathrm{R}$ stadsitics, discrimina dichas puntuaciones, eliminando las de menor significancia para el factor y presentando como resultados, solo aquellas que conforman dichos factores.

\section{DISCUSIÓN}

El objetivo del presente estudio fue analizar las posibles relaciones entre psicopatologías e ideación suicida; para lo cual, se exponen los análisis descriptivos de manera separada, las psicopatologías identificadas, el nivel de riesgo de ideación suicida y finalmente, los hallazgos sobre la correlación entre las variables de estudio, analizándolos a la luz de los antecedentes teóricos y empíricos.

En cuanto a la mayor presencia de síntomas de trastornos psicopatológicos, estos se relacionan con Quejas somáticas (SOM), Manía (MAN), Esquizofrenia 
(ESQ), Trastornos de ansiedad (TRA) en un $9 \%$ y Depresión (DEP), en un $8 \%$; estos datos difieren de lo reportado por la Encuesta Nacional de Salud Mental en la que se indica que las psicopatologías con mayor frecuencia en adolescentes corresponde a TDA-H, trastorno disocial y abuso de sustancias (Ministerio de Salud, 2015); no obstante, coincide con los hallazgos relacionados con los trastornos depresivos. Por su parte, se identifican similitudes con los hallazgos de Petersen, Brulin y Bergström (2006) quienes reportaron quejas somáticas en aproximadamente un tercio de niños y adolescentes entre los 8 y los 14 años de edad.

Adicionalmente, se encontró similitud en los índices arrojados para Tratarnos de Ansiedad (TRA) y Esquizofrenia (ESQ) correspondiente al $9 \%$, respecto a los hallazgos de Tayeh, Agámez \& Chaskel (2016) quienes refirieron que entre el 2,8 \% y $32 \%$ de los niños y adolescentes podrían presentar síntomas relacionados con ansiedad, y Beitia-Cardona, Rodríguez-Torres, Estrada-González y Benavides-Portilla (2019), quienes encontraron mayor prevalencia de esquizofrenia. Estos hallazgos, ponen de manifiesto la importancia de realizar acompañamiento de manera más cercana al proceso de desarrollo tanto físico como mental del adolescente, buscando identificar factores de riesgo, pues como se ha reportado, esta es una etapa de alta vulnerabilidad y por tanto, existe mayor riesgo de presencia de alguna psicopatología (Ministerio de Salud, 2015).

Respecto a la variable Ideación Suicida (IS), se identificó que el 48,5 \% de los estudiantes presentaron niveles alto y muy alto, este hallazgo concuerda con estudios desarrollados por Ventura-Juncá, Carvajal, Undurraga, Vicuña, Egaña y Garib (2010); Florenzano, Valdés, Cáceres, Santander, Aspillaga, y Musalem (2011) y Varengo (2016), quienes ya habían reportado su presencia entre el $28,6 \%$ y el $62 \%$ de los adolescentes entre 15 y 18 años, y de manera particular, se identificó presencia de Ideación Suicida Positiva (ISP) en el 51,9\% de los participantes y de Ideación Suicida Negativa (ISN) en el 30,1\%; dichas cifras concuerdan con los hallazgos de Forero, Siabato y Salamanca (2017) quienes encontraron que más del $28 \%$ presentaba puntuaciones elevadas en las tres subescalas del inventario de Ideación Suicida Positiva y Negativa. Teniendo en cuenta la alta manifestación de ideación suicida en población adolescente y si bien, la medida de evaluación inicial no necesariamente configura una conducta suicida, reconocer dicha manifestación como señal de alarma, permite generar medidas de prevención que apunten al fortalecimiento de las redes de apoyo.

Al analizar la posible relación entre las psicopatologías evaluadas y la ldeación Suicida (IS) mediante un análisis factorial, los resultados revelan dos factores 
que reúnen las psicopatologías asociadas a las subescalas de Ideación Suicida Positiva (ISP) e Ideación Suicida Negativa (ISN). El primer factor latente, compuesto por ocho psicopatologías (Quejas somáticas (SOM), Ansiedad (ANS), Trastornos relacionados con la ansiedad (TRA), Manía (MAN), Paranoia (PAR), Esquizofrenia (ESQ), Depresión (DEP) y Rasgos límites (LIM)), podría indicar que un individuo que presente patrones propios de una de estas psicopatologías, tendería a presentar déficits en factores protectores, tales como esperanza, autocontrol, satisfacción y autoeficacia.

De acuerdo con estos resultados, se considera importante resaltar los planteamientos de Corona, Hernández y García (2016), al referir que los factores protectores, disminuyen la probabilidad de que surja un trastorno mental, dentro de los que se destacan: buena salud, hábitos saludables, apoyo social y familiar, construcción de espacios públicos seguros, cobertura en salud, entre otros (OPS, 2011; Lomba, Alfonso y Fernández, 2014). Así mismo, se ha encontrado que la presencia de satisfacción con la vida y optimismo correlaciona negativamente con la psicopatología (Mustaca, Kamenetzky y Vera, 2010) y que el autocontrol se relaciona de manera inversa con la presencia de trastornos (Boals, Vandellen y Banks, 2011).

El segundo factor latente, incluye las psicopatologías Depresión (DEP) y Rasgos límites (LIM), y se relaciona con la subescala de Ideación Suicida Negativa (ISN); a partir de este, se podría indicar que quienes presentan sintomatología depresiva y/o rasgos límites, tenderían a presentar ideas relacionadas con el suicidio, este hallazgo concuerda con lo expuesto por Salamanca y Siabato (2017), quienes encontraron mayor proporción de estudio en los que relacionan dichas psicopatologías con la ideación suicida.

En cuanto a la relación entre LIM e ISN, esta puede estar asociada con una de las características de este trastorno, la cual corresponde a comportamientos suicidas incluyendo la ideación; por tanto y tal como se define en el Manual Diagnóstico y Estadístico de los Trastornos Mentales (APA, 2014) y lo evidencian los hallazgos de Espinosa, Grynberg y Romero (2009), quienes encontraron que el 93,3 \% de los pacientes diagnosticados con Trastorno Límite, manifiesta pensamientos suicidas, sugiriendo un alto riesgo de intento y consumo del suicidio. Esto a su vez, se puede atribuir a la inestabilidad emocional que presentan, la cual se caracteriza por una alta impulsividad y poco control; así como al establecimiento de relaciones problemáticas (Escribano, 2006).

Específicamente, la asociación encontrada entre Depresión (DEP) e Ideación Suicida (IS) $(0,46)$ concuerda con los hallazgos obtenidos por De la Torre, 
Cubillas, Pérez, y Valdez (2009), quienes identificaron que los participantes que tenían depresión, tenían un riesgo 8,8 veces mayor de presentar ideación suicida; de igual manera, Luján (2017) identificó que en un grupo con ideación suicida, sobresalía la depresión con un $56,3 \%$; lo anterior, puede explicarse debido a que los sentimientos de tristeza, soledad, desesperanza, anhedonia son síntomas propios a la depresión, por lo tanto, hacen vulnerable a los individuos para presentar Ideación Suicida (IS) (Toledo, 2012; Girard, 2017; Siabato, Forero y Salamanca, 2017).

Por su parte, la no relación entre las psicopatologías Rasgos antisociales (ANT), Problemas con el alcohol (ALC) y problemas con las Drogas (DRG) y, los factores de la Ideación Suicida (IS), difiere de los estudios realizados por el Servicio Andaluz de Salud (2010) y Bousoño, Al-Halabí, , Burón, Garrido, Díaz-Mesa, Galván, García- Álvarez, Carli, Hoven, Sarchiapone, Wasserman, Bousoño, García-Portilla, Iglesia, Sáiz y Bobes (2017), quienes encontraron relaciones importantes entre estas variables, específicamente, con el consumo de sustancias psicoactivas, lo cual supone un riesgo para presentar ideas suicidas (González-Forteza, Juárez, Montejo, Oseguera, Wagnery Jiménez, 2015); esta diferencia puede deberse a que pocos de los participantes reportaron síntomas relacionados con estas psicopatologías, probablemente relacionado con aspectos como la deseabilidad social.

De acuerdo a los resultados y teniendo en cuenta el objetivo de estudio, se concluye que existe una relación significativa $(r=0,37 ; p<0,05)$ entre los dos constructos, lo cual concuerda con estudios previos sobre el riesgo de suicidio para personas con enfermedades mentales, el cual aumenta de tres a 12 veces (Kaplan, Sadock \& Grebb, 1999). Asimismo, hay evidencia de estudios que se dedican a la relación de trastornos específicos con la Ideación Suicida (IS), encontrando correlaciones significativas; tal es el caso de Andrade (2012) quien, mediante una revisión documental, concluye que las tendencias suicidas se encuentran relacionadas con depresión, ansiedad e ideas obsesivo-compulsivas.

Por su parte, Silva, Vicente, Saldivia y Kohn (2013) identificaron relaciones importantes entre el trastorno psicótico, bipolaridad, trastorno depresivo, distimia, agorafobia, dependencia al alcohol, abuso y dependencia a drogas y trastorno por somatización e ideación suicida; Cromer, Villodas y Chou (2018) hallaron una relación significativa y positiva entre victimización acumulativa e Ideación Suicida (IS), siendo los síntomas depresivos los que median dicha relación durante la adolescencia, y González-Rodríguez, Molina-Andreu, Navarro, Gastó, Penadés y Catalán (2014), quienes evidenciaron en su revisión una relación entre paranoia e ideación suicida de 8 a 21 \%; que a 
su vez coincide con el estudio epidemiológico realizado por Mármol, Luque, Farouk y Fernández-Crehuet (2018).

Al respecto, es importante atender a los hallazgos referidos en la escala de Ideación Suicida Positiva (ISP) debido a que la mayoría de trastornos guardan relación con el déficit de factores protectores como esperanza, satisfacción, autoeficacia, autoestima y autocontrol, lo cual indica la relevancia de estudiar la ideación suicida no solo desde los pensamientos, deseos y planes suicidas sino también, desde la carencia de factores de protección, hallazgos que han sido soportados por Castro-Rueda, Martínez-Villalba, Camacho y RuedaJaimes (2010), al referir como factores protectores del riesgo suicida, las razones para vivir, las cuales generalmente se relacionan con el sentido de responsabilidad para con la familia. Por tanto, al analizar todas las variables en conjunto, a través del Análisis Factorial Confirmatorio, se encontraron las relaciones y significancia de estas, para las causas de Ideación Suicida debidas a psicopatologías.

Estos resultados además, aportan evidencia sobre los métodos estadísticos y en especial, sobre los métodos de ecuaciones estructurales, como herramientas para el análisis de estudios en temas de Psicología; demostrando, que son una buena alternativa para buscar relaciones entre variables medidas sobre un sujeto, cuando se trata de medir más de dos características, permitiendo conocer factores importantes de relación e ideando un perfil de los posibles diagnósticos; con el fin de generar estrategias de prevención a nivel universal, selectivo y específico en un contexto determinado.

\section{CONCLUSIONES}

Si bien la mayoría de los participantes se ubicaron en los niveles sin síntomas y bajo, respecto a la presencia de Psicopatologías, las relacionadas con quejas somáticas, manía, esquizofrenia, trastornos relacionados con ansiedad y depresión, fueron identificadas en un porcentaje entre el 9 y el $12 \%$; estos datos, concuerdan con las patologías más prevalentes, reportadas por la OMS (2018).

El 48,5\%, manifestó síntomas relacionados, siendo más predominantes los que tienen que ver con la carencia de factores protectores; aspectos que son resaltados por los hallazgos de quien refiere la importancia de variables asociadas a razones para vivir y en especial, el sentido de responsabilidad hacia la familia (Castro- Rueda, Martínez-Villalba, Camacho y Rueda- Jaimes, 2010). 
El Análisis Factorial, revela dos factores que reúnen ocho de las 11 psicopatologías evaluadas por el Inventario de Evaluación de la Personalidad para Adolescentes (PAI-A) y que se relacionan con los factores asociados a las subescalas de Ideación Suicida; el primer factor latente, reúne ocho psicopatologías (Quejas somáticas, Ansiedad, Trastornos relacionados con ansiedad, Manía, Paranoia, Esquizofrenia, Depresión y Rasgos límites), que se relacionan con la Ideación Suicida Positiva y el segundo factor latente (Depresión y Rasgos Límites), con Ideación Suicida Negativa.

Los resultados del presente estudio, además de corroborar los hallazgos previos, ponen de manifiesto la importancia de trabajar sobre la ausencia de los factores de protección, como elemento fundamental para el desarrollo de ideación suicida, además de identificar las características puntuales de cada Psicopatología y su relación directa con los factores de riesgo y protección de ideación suicida; sin embargo, se requiere contar con mayor soporte empírico en cuanto a la presencia del trastorno antisocial y el consumo de drogas y alcohol y, su relación con la ideación suicida.

Conflicto de interés: Los autores de este artículo declaran no tener conflictos de interés

Agradecimientos: A los investigadores Gonzalo Daniel Galvan Patrignani, Ph.D y Francisco Javier Vásquez De la Hoz, Mg., de la Universidad Cooperativa de Colombiasede Montería, por la gestión en la consecución del Inventario de Evaluación de la Personalidad (PAI-A) y el apoyo durante todo el proceso investigativo; al investigador Fredy Hernán Villalobos Galvis, Ph.D., de la Universidad de Nariño, por el préstamo del Inventario de Ideación Suicida Positiva y Negativa (PANSI) y a Camilo Ernesto Cadena Salinas, del semillero del grupo de investigación Clínica y Salud de la Universidad Pedagógica y tecnológica de Colombia, por el apoyo en la aplicación de instrumentos.

Financiamiento: Proyecto sin financiación, derivado del proyecto denominado Validación del Inventario de Evaluación de la Personalidad para adolescentes PAI-A (ID:1890) adscrito a la Universidad Cooperativa de Colombia- sede Montería, en alianza con la Universidad Cooperativa de Colombia - sedes Santa Marta y Barrancabermeja, la Universidad Pedagógica y Tecnológica de Colombia- sede Tunja y CECAR- sede Sincelejo.

\section{REFERENCIAS}

Andrade, J. A. (2012). Aspectos Psicosociales del Comportamiento Suicida en Adolescentes. Revista Electrónica de Psicología Iztacala, 15(2), 688-721. http://www. revistas.unam.mx/index.php/repi/article/view/32373

Asociación Americana de Psiquiatría. (2014). Trastornos de la personalidad. En Manual Diagnóstico y estadístico de trastornos mentales (5aed.). España: Editorial Médica Panamericana https://doi.org/10.1176/appi.books.9780890425596. dsm05 
Bedoya, E. Y. y Montaño, L. E. (2016). Suicidio y trastorno mental. Revista CES. Psicología, 9(2), 179-201. http://dx.doi.org/10.21615/cesp.9.2.12

Beitia-Cardona, P., Rodríguez-Torres, E., Estrada-González, C., y Benavides-Portilla, M. (2019). Intento suicida y factores asociados en dos instituciones de CaliColombia. Revista Ciencia y Cuidado, 16(1), 19-31. https://revistas.ufps.edu.co/ index.php/cienciaycuidado/article/view/1542

Boals, A., Vandellen, M. R., \& Banks, J. B. (2011). The relationship between self-control and health: The mediating effect of avoidant coping. Psychology \& Health, 26(8), 1049-1062. https://psycnet.apa.org/record/2011-19622-007

Bousoño, M. S., Al-Halabí, S., Burón, P., Garrido, M., Díaz-Mesa, E. M., Galván, G., García- Álvarez, L., Carli, V., Hoven, C., Sarchiapone, M., Wasserman, D., Bousoño, M., García-Portilla, M. P., Iglesia, C., Sáiz, P. A. y Bobes, J. (2017). Uso y abuso de sustancias psicotrópicas e internet, psicopatología e ideación suicida en adolescentes. Adicciones, 29(2), 97-104. https://medes.com/publication/121745

Cabra, O., Infante, D. y Sossa, F. (2010). El suicidio y los factores de riesgo asociados en niños y adolescentes. Revista Médica, Sanitas, 13(2), 28-35. http://www. unisanitas.edu.co/Revista/18/suicidio.pdf

Castro-Rueda, V., Martínez-Villalba, A Camacho, P y Rueda-Jaimes, G. (2010). Factores de riesgo y protección para Intento Suicida en adultos colombianos con suicidabilidad. Revista Colombiana de Psiquiatría, 39(4), 705- 715.

Cardenal, V., Ortiz-Tallo, M. y Santamaría, P. (2012). PAI-A. Inventario de evaluación de la personalidad para adolescentes. Manual de aplicación-Versión experimental. Madrid: Tea Ediciones.

Carvajal, G., y Caro, C. V. (2011). Ideación suicida en la adolescencia: una explicación desde tres de sus variables asociadas en Bogotá, 2009. Colombia Médica, 42(2), 45-56. http://www.bioline.org.br/pdf?rc11039

Ceballos-Ospino, G. A., Suárez-Colorado, Y., Suescún-Arregocés, J., Gamarra-Vega, L. M., González, K. E., \& Sotelo-Manjarres, A. P. (2015). Ideación suicida, depresión y autoestima en adolescentes escolares de Santa Marta. Duazary, 12(1), 15-22. https://revistas.unimagdalena.edu.co/index.php/duazary/article/view/1394

Corona, B., Hernández, M. y García, R. (2016). Mortalidad por suicidio, factores de riesgos y protectores. Revista Habanera de Ciencias Médicas, 15(1) http:// scielo.sld.cu/scielo.php?script=sci_arttext\&pid=S1729-519X2016000100011\&ln$\mathrm{g}=\mathrm{es} \& \mathrm{t} \operatorname{lng}=\mathrm{es}$.

Cromer, K. D., Villodas, M. T., y Chou, T. (2018). Gender differences in psychological distress as a mediational pathway to suicidal ideation among adolescents at high risk for victimization by violence. Psychology of Violence. Advance online publication. https://psycnet.apa.org/search/display?id=d60e9e82-c8ce-ddad-22ba-05cf9e$84 \mathrm{fb} 50 \&$ recordld $=1 \&$ tab $=$ PA\&page $=1 \&$ display $=25 \&$ sort $=$ Publication YearMSSort\%20desc,AuthorSort\%20asc\&sr=1

De la Torre, I. M., Cubillas, M. J., Pérez, R., y Valdez, E. (2009). Ideación suicida en población escolarizadainfantil:factorespsicológicosasociados.SaludMental,32(1),495-502.http:// www.scielo.org.mx/scielo.php?script=sci_arttext\&pid=\$0185-33252009000600007

Escribano, T. (2006). Trastorno Límite de la Personalidad: Estudio y Tratamiento. Intelligo, 1(1), 4-20. https://www.trastornolimite.com/images/stories/pdf/TLP-estudio-y-tratamiento-por-tania-escribano-nieto.pdf

Espinosa, J. J., Grynberg, B. B. y Romero, M. P. (2009). Riesgo y letalidad suicida en pacientes con trastorno límite de la personalidad (TLP), en un hospital de psiquiatría. Salud Mental, 32, 317-325. http://www.scielo.org.mx/scielo.php?script=sci_arttext\&pid=S0185-33252009000400007 
Florenzano, R., Valdés, M., Cáceres, E., Santander, S., Aspillaga, C., y Musalem, C. (2011). Relación entre ideación suicida y estilos parentales en un grupo de adolescentes chilenos. Revista médica de Chile, 139(12), 1529-1533. https:// scielo.conicyt.cl/scielo.php?script=sci_arttext\&pid=S0034-98872011001200001

Flores, N., Perales, Y. y Gómez, W. (2016). Depresión e ideación suicida en los adolescentes de una Institución Educativa Pública del distrito de Chongos Bajo - Perú 2014. Ágora Revista Científica, 3(1), 265-274. https://revistaagora.com/index. php/cieUMA/article/viewFile/50/54

Forero, I., Siabato, E. y Salamanca, Y. (2017). Ideación suicida, funcionalidad familiar y consumo de alcohol en adolescentes de Colombia. Revista Latinoamericana de Ciencias Sociales, Niñez y Juventud, 15(1), 431-442. http://www.scielo.org.co/ scielo.php?pid=S1692-715X2017000100028\&script=sci_abstract\&tlng=es

Girard, G. (2017). El suicidio en la adolescencia y en la juventud. Revista de Formación Continuada de la Sociedad Española de Medicina de la Adolescencia, 5(2), 22-31. https://www.adolescenciasema.org/ficheros/REVISTA\%20ADOLESCERE/ vol5num2-2017/22-31.pdf

Gómez, A (2012). Evaluación del riesgo de suicidio: enfoque actualizado. Revista Médica Clínica las Condes, 23(5), 607- 615.

González-Forteza, C., Juárez, C. E., Montejo, L. A., Oseguera, G., Wagner, F. A. y Jiménez, A. (2015). Ideación suicida y su asociación con drogas, depresión e impulsividad en una muestra representativa de estudiantes de secundaria del estado de Campeche, México. Acta Universitaria, 25(2), 29-34. http://omextad. salud.gob.mx/contenidos/noticias/Ideacion.pdf

González-Rodríguez, A., Molina-Andreu, O., Navarro, V., Gastó, C., Penadés, R. y Catalán, R. (2014). Delusional disorder: no gender differences in age at onset, suicidal ideation, or suicidal behavior. Revista Brasileira de Psiquiatría, 36(2), 119-124. www.scielo.br/scielo.php?script=sci_arttext\&pi$\mathrm{d}=$ S1516-44462014000200119\&Ing=en\&tIng=en

Instituto Nacional de Medicina Legal y Ciencias Forenses (2018). Boletín estadístico mensual cifras enero-diciembre de 2018. www.medicinalegal.gov.co/cifras-estadisticas/forensis

Hernández, R., Fernández-Collado, C. y Baptista, P. (2010). Metodología de la investigación. México: McGrawHill.

Kaplan, H., Sadock, B. y Grebb, J. (1999). Sinopsis de Psiquiatría. Ciencias de la conducta Psiquiatría clínica. Buenos Aires, Argentina: Editorial Médica Panamerica.

Lomba Acevedo, P., Alfonso Carrillo, E. y Fernández Coto, L. (2013). “Prevención del suicidio en la comunidad". En Medio Residencial y Salud. La Habana: Consejo editorial OMS y OPS; pp. 207-214.

Luján La Torre, M. A. (2017). Trastornos clínicos de personalidad en estudiantes de psicología que manifiestan ideación suicida/intento suicida. (Tesis de Grado). Universidad Nacional de San Agustín de Arequipa, Perú. https://alicia.concytec. gob.pe/vufind/Record/UNSA_d08b5e16808e078aa4ca5a664f69c5ce/Description\#tabnav

Mármol, F., Luque, R., Farouk, M. y Fernández-Crehuet, R. (2018). Trastorno delirante y conducta suicida: estudio descriptivo de 26 casos. Revista Chilena de Neuro-Psiquiatría, 56(4), 228- 240. https://scielo.conicyt.cl/scielo.php?script=sci_arttext\&pid=S0717-92272018000400228

Ministerio de Salud (2015). Encuesta Nacional de Salud Mental Colombia. Protocolo del estudio. Revista Colombiana de Psiquiatría, 45, 2-8. https://www.elsevier. es/es-revista-revista-colombiana-psiquiatria-379-articulo-encuesta-nacional-salud-mental-colombia-S0034745016300312 
Ministerio de Salud (2018). Boletín de salud mental. Análisis de Indicadores en Salud Mental por territorio. https://www.minsalud.gov.co/sites/rid/Lists/BibliotecaDigital/RIDE/VS/PP/ENT/boletin-onsm-abril-2018.pdf.

Morey, L. C. (2007). Personality Assessment Inventory-Adolescent (PAI-A). Lutz, FL: Psychological Assessment Resources.

Mustaca, A. E., Kamenetzky, G. y Vera, P. (2010). Relaciones entre Variables Positivas y Negativas en una Muestra de Estudiantes Argentinos. Revista Argentina de Clínica Psicológica, 19(1), 227-235. https://www.redalyc.org/pdf/2819/281921798004. pdf

Nizama, M. (2011). Suicidio. Revista Peruana de Epidemiología, 15(2), 81-85. https:// www.redalyc.org/pdf/2031/203122516002.pdf

Nock, M. K., Borges, G., Bromet, E. J., Alonso, J., Angermeyer, M., Beautrais, A., Bruffaerts, R., Chiu, W. T., de Girolamo, G., Gluzman, S., de Graaf, R., Gureje, O., Haro, J. M., Huang, Y., Karam, E., Kessler, R. C., Lepine, J. P., Levinson, D., Medina-Mora, M. E., Ono, Y., Posada-Villa, J. y Williams, D. (2008). Cross-national prevalence and risk factors for suicidal ideation, plans and attempts. The British journal of psychiatry: the journal of mental science, 192(2), 98-105. https://doi. org/10.1192/bjp.bp.107.040113

Organización Mundial de la Salud (2017). Suicidio, nota descriptiva. http://www.who. int/mediacentre/factsheets/fs398/es/

Organización Mundial de la Salud (2018). La carga de los trastornos mentales en la Región de las Américas. https://consultorsalud.com/wp-content/ uploads/2019/01/la_carga_de_los_trastornos_mentales_en_la_region_de_las_ americas_2018_.pdf

Organización Panamericana de la Salud (OPS). (2011). Marco de Referencia para la implantación de la Estrategia Regional de Salud Mental. Washington (DC): pp. 142-37

Osman, A., Gutiérrez, P. M., Kipper, B. A., Barrios, F. X. y Chiros, C. E. (1998). The positive and negative suicide ideation inventory: development and validation. Psychological Reports, 82, 783-793. https://journals.sagepub.com/doi/10.2466/ pro.1998.82.3.783

Pérez, N. y Salamanca, Y., (2017). Relación entre autoestima e ideación suicida en adolescentes colombianos. Revista de Psicología GEPU, 8(1), 8-21. https:// revistadepsicologiagepu.es.tl/Relaci\%F3n-entre-Autoestima-e-Ideaci\%F3n-Suicida-en-Adolescentes-Colombianos.htm

Petersen, S., Brulin, C., \& Bergström, E. (2006). Recurrent pain symptoms in young schoolchildren are often multiple. Pain, 121(1-2), 145-150. https://psycnet.apa. org/record/2006-02624-013

Ridge, A., Keyes, G. M., \& Jobes, D. A. (2016). Understanding and treating suicidal risk in young children. Practice Innovations, 1(1), 3-19. https://psycnet.apa.org/ record/2016-14272-002

Rodríguez, M., Rincón, H., Velasco, M., Hernández, C., Ramos, M. y González, J. (2012). Prevalencia de trastornos mentales en una población que demanda servicios de salud en una IPS de baja complejidad y su asociación con la ideación suicida la y discapacidad percibida. Revista Facultad Nacional de Salud Pública, 30(2), 141-151. http://www.scielo.org.co/pdf/rfnsp/v30n2/v30n2a03.pdf

Rosales, J. C. (2010). La investigación del proceso suicida. En L. L. Eguiluz, M. H. Córdova y J. C. Rosales (Eds.), Ante el suicidio. Su comprensión y tratamiento (pp. 81-94). México, D.F: Editorial Pax. 
Salamanca, Y. \& Siabato, E. (2017). Investigaciones sobre ideación suicida en Colombia, 2010-2016. Pensando Psicología, 13(21), 59-70. https://revistas.ucc. edu.co/index.php/pe/article/view/1714

Servicio Andaluz de Salud (2010). Recomendaciones sobre detección, prevención e intervención de la conducta suicida. https://consaludmental.org/publicaciones/ Recomendacionesprevencionsuicidio.pdf

Siabato Macías, E. F., Forero Mendoza, I. X., \& Salamanca Camargo, Y. (2017). Asociación entre depresión e ideación suicida en un grupo de adolescentes colombianos. Pensamiento psicológico, 15(1), 51-61. https://psycnet.apa.org/ record/2017-31535-004

Silva, D., Vicente, B., Saldivia, S. \& Kohn, R. (2013). Conducta suicida y trastornos psiquiátricos en Chile, un estudio poblacional. Revista médica de Chile, 141(10), 1275-1282. https://scielo.conicyt.cl/scielo.php?script=sci_arttext\&pid=S0034-98872013001000006

Toledo, A. M. (2012). El suicidio en el anciano institucionalizado. Revista Argentina de Clínica Neuropsiquiátrica, 17(2), 162-168. www.alcmeon.com.ar/17/66_ oct11/07_moreno_alc66.pdf

Varengo, J. (2016). Ideación Suicida en Adolescentes (Tesis de Grado). Universidad siglo 21 la educación evoluciona, Argentina. https://es.scribd.com/ document/416363018/ejemplo-de-suicidio

Vásquez, F., Galván, G., Guerrero, M., Simancas, M., Romero-Acosta, K., Ramírez, A., Yañez, H., Ferrel, F., Ferrel, L., Galván, F., y Salamanca, Y (2018). Estandarización para Colombia. En Morey, L.C. Baremos del PAI-A Inventario de Evaluación de la Personalidad para Adolescentes. España: TEA Ediciones, 10-15.

Ventura-Juncá, R., Carvajal, C., Undurraga, S., Vicuña, P., Egaña, J. y Garib, M. J. (2010). Prevalencia de ideación e intento suicida en adolescentes de la Región Metropolitana de Santiago de Chile. Rev Me, 138(1), 309-315. https://studylib. es/doc/6206215/prevalencia-de-ideación-e-intento-suicida-en-adolescentes

Villalobos-Galvis, T. (2009). Validez y fiabilidad del Inventario de Ideación Suicida Positiva y Negativa - PANSI en estudiantes colombianos. Universitas Psychologica, 9(2), 509-520. https://psycnet.apa.org/record/2010-14439-017

Zamora, R. \& Esnaola, J. (2015). Guía de trabajo en " $R$ ": Análisis factorial y análisis de componentes principales. Ayudantía Estadística IV, Facultad de Sociología, Universidad de Chile. https://docplayer.es/41799282-Guia-de-trabajoen-r.html 